\title{
SOME NOTES ON GEOMETRY OF BÉZIER CURVES IN EUCLIDEAN 4-SPACE
}

\author{
Esra Erkan $^{a^{*}(\mathbb{D})}$, Salim Yüce ${ }^{\mathrm{b}}$ \\ a Department of Mathematics, Faculty of Arts and Sciences, Harran University, Sanliurfa, Turkey \\ esraerkan@harran.edu.tr (*corresponding author) \\ ${ }^{b}$ Department of Mathematics Faculty, Faculty of Arts and Sciences, \\ Yildiz Technical University, Istanbul, Turkey \\ sayuce@yildiz.edu.tr
}

\begin{abstract}
The main purpose of this paper is to investigate Bézier curves in the Euclidean space $E^{4}$ with respect to differential geometry. For this purpose, the Serret-Frenet elements at every point, starting point and ending points are computed.
\end{abstract}

Keywords: Bézier curve, Serret-Frenet frame, triple (ternary) product

\section{Introduction}

Bézier curves are parametric smooth functions with significant aspects. There exist precious applications of this kind of curves in computer science, graphics, animations, modelling and many other related fields of engineering.

A Bézier curve is defined with the aid of control points. These points are ordered set of points $b_{0}, b_{1}, \ldots, b_{n}$ which provide the approximation for desired curve. A Bézier curve of degree $n$ with $(n+1)$ control points in Euclidean $n$-space $E^{n}$ is defined by [1].

$$
P(t)=\sum_{i=0}^{n} B_{i, n}(t) b_{i}, \quad 0 \leq t \leq 1
$$

Here, the functions $B_{i, n}(t)$ are Bernstein polynomials given by 
$B_{i, n}(t)=\left(\begin{array}{c}n \\ i\end{array}\right) t^{i}(1-t)^{n-i}$

where $\left(\begin{array}{l}n \\ i\end{array}\right)$ denotes $i^{\text {th }}$ binomial coefficient [2]. Considering the Bernstein polynomials derivatives, the $r^{\text {th }}$-order derivative of the Bézier curve of degree $n$ is represented by

$$
P^{(r)}(t)=\frac{n !}{(n-r) !} \sum_{i=0}^{n-r} B_{i, n-r}(t) \Delta^{r} b_{i}
$$

where $\Delta^{r} b_{i}$ is the difference equation indicated by $\Delta^{r} b_{i}=\Delta^{r-1} b_{i+1}-\Delta^{r-1} b_{i} \quad[3,4]$.

Furthermore, the $r^{\text {th }}$-order derivative of a Bézier curve of degree $n$ at starting and ending points is represented by the following equations, respectively [3]:

$\left.P^{(r)}(t)\right|_{t=0}=\frac{n !}{(n-r) !} \Delta^{r} b_{0}$

and

$\left.P^{(r)}(t)\right|_{t=1}=\frac{n !}{(n-r) !} \Delta^{r} b_{n-r}$

Taking into consideration (2), (3) and (4), various authors studied the geometric properties of Bézier curves. In [5], K. Kenmotsu investigated surfaces of revolution with a periodic mean curvature function and their visualization, G. H. Georgiev computed shape curvatures of cubic Bézier curves in [6], H. Kuşak Samanc1, S. Çelik and M. İncesu [7] obtained the Bishop frame of Bezier curves in Euclidean 3- space and the authors [8] presented the Serret-Frenet elements of these kind of curves in any Euclidean plane and Euclidean 3-space etc.

Motivated by these facts, we study Bézier curves in Euclidean 4-space and we obtain the Serret-Frenet frame of these curves. By the help of this frame, we compute the principle curvatures of Bézier curves in Euclidean 4-space.

\section{Basic concepts in four dimensional space}

In this section, we shall recall some general definitions and basic formulas dealing the standard real vector space $\mathbb{R}^{4}$ and Euclidean 4 -space.

\subsection{Ternary product}

We begin this subsection with the following definition:

Definition 2.1 Let $\left\{e_{1}, e_{2}, e_{3}, e_{4}\right\}$ be the standard basis of $\mathbb{R}^{4}$ and $x=\sum_{j=1}^{4} x_{j} e_{j}, y=\sum_{j=1}^{4} y_{j} e_{j}$ and $z=\sum_{j=1}^{4} z_{j} e_{j}$ be any three linear independent vectors in $\mathbb{R}^{4}$. The ternary product (or triple vectorial product) of these vectors is defined by 
$x \otimes y \otimes z=\left|\begin{array}{cccc}e_{1} & e_{2} & e_{3} & e_{4} \\ x_{1} & x_{2} & x_{3} & x_{4} \\ y_{1} & y_{2} & y_{3} & y_{4} \\ z_{1} & z_{2} & z_{3} & z_{4}\end{array}\right|$

and the following relations satisfy

$x \otimes y \otimes z=y \otimes z \otimes x=z \otimes x \otimes y=-(z \otimes y \otimes x)=-(y \otimes x \otimes z)=-(x \otimes z \otimes y)$ [9], [10].

Furthermore, we have the following algebraic properties of ternary product for each $a, b, c, d, e, f, x, y, z \in \mathbb{R}^{4}$ and $\lambda, \mu \in \mathbb{R},[10,11]$

(1) $(x+y) \otimes(z \otimes t)=(x \otimes z \otimes t)+(y \otimes z \otimes t)$

(2) $(a+\lambda b+\mu c) \otimes b \otimes c=a \otimes b \otimes c$

(3) $x \otimes y \otimes z=0 \Leftrightarrow x, y$ and $z$ are linearly dependent.

(4) $\langle x, y \otimes z \otimes t\rangle=\operatorname{det}(x, y, z, t)=\langle x \otimes y \otimes z, t\rangle$ (quadruple scalar product)

(5) $\langle x, x \otimes y \otimes z\rangle=\langle y, x \otimes y \otimes z\rangle=\langle z, x \otimes y \otimes z\rangle=0$.

(6) $d \otimes e \otimes(a \otimes b \otimes c)=\left|\begin{array}{ccc}a & b & c \\ \langle a, e\rangle & \langle b, e\rangle & \langle c, e\rangle \\ \langle a, d\rangle & \langle b, d\rangle & \langle c, d\rangle\end{array}\right|$.

is stated the quintuple vector product of vectors $a, b, c, d$ and $e$ and a linear combination of vectors $a, b$ and $c$. It can be interpreted as a vector lying in the space of vectors $a, b$ and $c$.

$(7) a \otimes(d \otimes e \otimes f) \otimes(x \otimes y \otimes z)=\left|\begin{array}{ccc}\langle a, x\rangle & \langle a, y\rangle & \langle a, z\rangle \\ \langle d \otimes e \otimes f, x\rangle & \langle d \otimes e \otimes f, y\rangle & \langle d \otimes e \otimes f, z\rangle \\ x & y & z\end{array}\right|$.

(8) $\left\|a_{1} \otimes a_{2} \otimes a_{3}\right\|^{2}=\Delta\left(a_{1}, a_{2}, a_{3}\right)=\left|\begin{array}{lll}\left\langle a_{1}, a_{1}\right\rangle & \left\langle a_{1}, a_{2}\right\rangle & \left\langle a_{1}, a_{3}\right\rangle \\ \left\langle a_{2}, a_{1}\right\rangle & \left\langle a_{2}, a_{2}\right\rangle & \left\langle a_{2}, a_{3}\right\rangle \\ \left\langle a_{3}, a_{1}\right\rangle & \left\langle a_{3}, a_{2}\right\rangle & \left\langle a_{3}, a_{3}\right\rangle\end{array}\right|$.

Here, $\Delta\left(a_{1}, a_{2}, a_{3}\right)$ denotes the $p \times p$ Gram determinant is, namely, $a_{i j}=\left\langle a_{i}, a_{j}\right\rangle, i, j=1, \ldots, p$.

\subsection{The theory of curves}

Let $\beta: I \subset \mathbb{R} \rightarrow E^{4}, \beta(t)=\left(\beta_{1}(t), \beta_{2}(t), \beta_{3}(t), \beta_{4}(t)\right)$ be a regular curve in Euclidean space $E^{4}$. We shall use the notation $\frac{d \beta}{d s}=\beta^{\prime}$ for the arc-length parameter $s$ and use the notation $\frac{d \beta}{d s}=\dot{\beta}$ for arbitrary parameter $s$ throughout the paper.

Now we recall the following theorems of [12]. 
Theorem 2.2 Frenet frame vectors $\left\{T, N, B_{1}, B_{2}\right\}$ of a regular curve $\beta$ in $E^{4}$ are given by the following formulas:

$$
\begin{aligned}
& T(t)=\frac{\dot{\beta}(t)}{\|\dot{\beta}(t)\|}, \\
& N(t)=\frac{B_{1}(t) \otimes B_{2}(t) \otimes \dot{\beta}(t)}{\left\|B_{1}(t) \otimes B_{2}(t) \otimes \dot{\beta}(t)\right\|}, \\
& B_{1}(t)=\frac{B_{2}(t) \otimes \dot{\beta}(t) \otimes \ddot{\beta}(t)}{\left\|B_{2}(t) \otimes \dot{\beta}(t) \otimes \ddot{\beta}(t)\right\|}, \\
& B_{2}(t)=\frac{\dot{\beta}(t) \otimes \ddot{\beta}(t) \otimes \dddot{\beta}(t)}{\|\dot{\beta}(t) \otimes \ddot{\beta}(t) \otimes \ddot{\beta}(t)\|} .
\end{aligned}
$$

Theorem 2.3 The first, second and third curvatures of the curve $\beta$ are given respectively by

$$
\begin{aligned}
& \kappa_{1}(t)=\frac{\langle N(t), \ddot{\beta}(t)\rangle}{\|\dot{\beta}(t)\|^{2}} \\
& \kappa_{2}(t)=\frac{\left\langle B_{1}(t), \dddot{\beta}(t)\right\rangle}{\|\dot{\beta}(t)\|^{3} \kappa_{1}(t)}, \\
& \kappa_{3}(t)=\frac{\left\langle B_{2}(t), \beta^{(4)}(t)\right\rangle}{\|\dot{\beta}(t)\|^{4} \kappa_{1}(t) \kappa_{2}(t)} .
\end{aligned}
$$

\section{Serret-Frenet elements of Bézier curve of degree $n$ in Euclidean space $E^{4}$}

Let $b_{0}, b_{1}, b_{2}, \ldots, b_{n}$ be non-linear $(n+1)$ - points in $E^{4}$ and $P(t)$ be a non-unit speed Bézier curve of degree $n$ with control points $b_{0}, b_{1}, b_{2}, \ldots, b_{n}$ in Euclidean space $E^{4}$ that is given by the following parametric equation

$$
P(t)=\sum_{i=0}^{n} B_{i, n}(t) b_{i}=B_{0, n}(t) b_{0}+B_{1, n}(t) b_{1}+\ldots+B_{n, n}(t) b_{n}
$$

Then, we obtain the following theorem:

Theorem 3.1 The Serret-Frenet frame $\left\{T, N, B_{1}, B_{2}\right\}$ of the curve $P(t)$ defined with (12) is given by

$$
T(t)=\frac{\sum_{i=0}^{n-1} B_{i, n-1}(t) \Delta b_{i}}{\left\|\sum_{i=0}^{n-1} B_{i, n-1}(t) \Delta b_{i}\right\|},
$$




$$
\begin{aligned}
& N(t)=\frac{\sum_{i=0}^{n-1} \sum_{j=0}^{n-2} \sum_{k=0}^{n-3} B_{i, n-1}^{4}(t) B_{j, n-2}^{3}(t) B_{k, n-3}^{2}(t)\left\{\begin{array}{l}
\left\{\left(\Delta b_{i} \otimes \Delta^{2} b_{j} \otimes \Delta^{3} b_{k}\right) \otimes \Delta b_{i} \otimes \Delta^{2} b_{j}\right\} \\
\otimes\left(\Delta b_{i} \otimes \Delta^{2} b_{j} \otimes \Delta^{3} b_{k}\right) \otimes \Delta b_{i}
\end{array}\right\}}{\left\|\sum_{i=0}^{n-1} \sum_{j=0}^{n-2} \sum_{k=0}^{n-3} B_{i, n-1}^{4}(t) B_{j, n-2}^{3}(t) B_{k, n-3}^{2}(t)\left\{\begin{array}{l}
\left\{\left(\Delta b_{i} \otimes \Delta^{2} b_{j} \otimes \Delta^{3} b_{k}\right) \otimes \Delta b_{i} \otimes \Delta^{2} b_{j}\right\} \\
\otimes\left(\Delta b_{i} \otimes \Delta^{2} b_{j} \otimes \Delta^{3} b_{k}\right) \otimes \Delta b_{i}
\end{array}\right\}\right\|}, \\
& B_{1}(t)=\frac{\sum_{i=0}^{n-1} \sum_{j=0}^{n-2} \sum_{k=0}^{n-3} B_{i, n-1}^{2}(t) B_{j, n-2}^{2}(t) B_{k, n-3}(t)\left\{\begin{array}{l}
\left(\Delta b_{i} \otimes \Delta^{2} b_{j} \otimes \Delta^{3} b_{k}\right) \\
\otimes \Delta b_{i} \otimes \Delta^{2} b_{j}
\end{array}\right\}}{\left\|\sum_{i=0}^{n-1} \sum_{j=0}^{n-2} \sum_{k=0}^{n-3} B_{i, n-1}^{2}(t) B_{j, n-2}^{2}(t) B_{k, n-3}(t)\left\{\begin{array}{l}
\left(\Delta b_{i} \otimes \Delta^{2} b_{j} \otimes \Delta^{3} b_{k}\right) \\
\otimes \Delta b_{i} \otimes \Delta^{2} b_{j}
\end{array}\right\}\right\|}, \\
& B_{2}(t)=\frac{\sum_{i=0}^{n-1} \sum_{j=0}^{n-2} \sum_{k=0}^{n-3} B_{i, n-1}(t) B_{j, n-2}(t) B_{k, n-3}(t)\left(\Delta b_{i} \otimes \Delta^{2} b_{j} \otimes \Delta^{3} b_{k}\right)}{\left\|\sum_{i=0}^{n-1} \sum_{j=0}^{n-2} \sum_{k=0}^{n-3} B_{i, n-1}(t) B_{j, n-2}(t) B_{k, n-3}(t)\left(\Delta b_{i} \otimes \Delta^{2} b_{j} \otimes \Delta^{3} b_{k}\right)\right\|} .
\end{aligned}
$$

for all $t \in \mathbb{R}$.

Proof By the equation (2), the first, second and third derivatives of $P(t)$ are found as follows:

$$
\begin{aligned}
& P^{\prime}(t)=n \sum_{i=0}^{n-1} B_{i, n-1}(t) \Delta b_{i}, \\
& P^{\prime \prime}(t)=n(n-1) \sum_{i=0}^{n-2} B_{i, n-2}(t) \Delta^{2} b_{i} \\
& P^{\prime \prime \prime}(t)=n(n-1)(n-2) \sum_{i=0}^{n-3} B_{i, n-3}(t) \Delta^{3} b_{i}
\end{aligned}
$$

Taking into account of (5) and (17), we have

$$
T(t)=\frac{P^{\prime}(t)}{\left\|P^{\prime}(t)\right\|}=\frac{n \sum_{i=0}^{n-1} B_{i, n-1}(t) \Delta b_{i}}{\left\|n \sum_{i=0}^{n-1} B_{i, n-1}(t) \Delta b_{i}\right\|}=\frac{\sum_{i=0}^{n-1} B_{i, n-1}(t) \Delta b_{i}}{\left\|\sum_{i=0}^{n-1} B_{i, n-1}(t) \Delta b_{i}\right\|} .
$$

Thus, we obtain the equation (13). Using (8), (17), (18), (19) and by a straightforward computation, we get

$$
B_{2}(t)=\frac{P^{\prime}(t) \otimes P^{\prime \prime}(t) \otimes P^{\prime \prime \prime}(t)}{\left\|P^{\prime}(t) \otimes P^{\prime \prime}(t) \otimes P^{\prime \prime \prime}(t)\right\|}=\frac{\sum_{i=0}^{n-1} \sum_{j=0}^{n-2} \sum_{k=0}^{n-3} B_{i, n-1}(t) B_{j, n-2}(t) B_{k, n-3}(t)\left(\Delta b_{i} \otimes \Delta^{2} b_{j} \otimes \Delta^{3} b_{k}\right)}{\left\|\sum_{i=0}^{n-1} \sum_{j=0}^{n-2} \sum_{k=0}^{n-3} B_{i, n-1}(t) B_{j, n-2}(t) B_{k, n-3}(t)\left(\Delta b_{i} \otimes \Delta^{2} b_{j} \otimes \Delta^{3} b_{k}\right)\right\|} .
$$

Thus, we obtain the equation (14). With a similar computations, we obtain 


$$
B_{1}(t)=\frac{B_{2}(t) \otimes P^{\prime}(t) \otimes P^{\prime \prime}(t)}{\left\|B_{2}(t) \otimes P^{\prime}(t) \otimes P^{\prime \prime}(t)\right\|}=\frac{\sum_{i=0}^{n-1} \sum_{j=0}^{n-2} \sum_{k=0}^{n-3} B_{i, n-1}^{2}(t) B_{j, n-2}^{2}(t) B_{k, n-3}(t)\left\{\begin{array}{l}
\left(\Delta b_{i} \otimes \Delta^{2} b_{j} \otimes \Delta^{3} b_{k}\right) \\
\otimes \Delta b_{i} \otimes \Delta^{2} b_{j}
\end{array}\right\}}{\left\|\sum_{i=0}^{n-1} \sum_{j=0}^{n-2} \sum_{k=0}^{n-3} B_{i, n-1}^{2}(t) B_{j, n-2}^{2}(t) B_{k, n-3}(t)\left\{\begin{array}{l}
\left(\Delta b_{i} \otimes \Delta^{2} b_{j} \otimes \Delta^{3} b_{k}\right) \\
\otimes \Delta b_{i} \otimes \Delta^{2} b_{j}
\end{array}\right\}\right\|}
$$

and

$$
N(t)=\frac{\sum_{i=0}^{n-1} \sum_{j=0}^{n-2} \sum_{k=0}^{n-3} B_{i, n-1}^{4}(t) B_{j, n-2}^{3}(t) B_{k, n-3}^{2}(t)\left\{\begin{array}{l}
\left\{\left(\Delta b_{i} \otimes \Delta^{2} b_{j} \otimes \Delta^{3} b_{k}\right) \otimes \Delta b_{i} \otimes \Delta^{2} b_{j}\right\} \\
\otimes\left(\Delta b_{i} \otimes \Delta^{2} b_{j} \otimes \Delta^{3} b_{k}\right) \otimes \Delta^{1} b_{i}
\end{array}\right\}}{\left\|\sum_{i=0}^{n-1} \sum_{j=0}^{n-2} \sum_{k=0}^{n-3} B_{i, n-1}^{4}(t) B_{j, n-2}^{3}(t) B_{k, n-3}^{2}(t)\left\{\begin{array}{l}
\left\{\left(\Delta b_{i} \otimes \Delta^{2} b_{j} \otimes \Delta^{3} b_{k}\right) \otimes \Delta b_{i} \otimes \Delta^{2} b_{j}\right\} \\
\otimes\left(\Delta b_{i} \otimes \Delta^{2} b_{j} \otimes \Delta^{3} b_{k}\right) \otimes \Delta b_{i}
\end{array}\right\}\right\|} .
$$

This completes the proof of theorem.

Theorem 3.2 The first, second and third curvatures $\kappa_{1}, \kappa_{2}, \kappa_{3}$ of $P(t)$ defined with (12) are given, respectively, by the following formulas:

$\kappa_{1}(t)=\frac{n-1}{n} \frac{\left.\sum_{i=0}^{n-1} \sum_{j=0}^{n-2} \sum_{k=0}^{n-3} B_{i, n-1}^{4}(t) B_{j, n-2}^{4}(t) B_{k, n-3}^{2}(t)\left\{\begin{array}{l}\left\{\begin{array}{l}\left\{\left(\Delta b_{i} \otimes \Delta^{2} b_{j} \otimes \Delta^{3} b_{k}\right) \otimes \Delta b_{i} \otimes \Delta^{2} b_{j}\right\} \\ \left(\Delta b_{i} \otimes \Delta^{2} b_{j} \otimes \Delta^{3} b_{k}\right) \otimes \Delta b_{i}\end{array}\right.\end{array}\right\}, \Delta^{2} b_{j}\right\rangle}{\left\|\sum_{i=0}^{n-1} \sum_{j=0}^{n-2} \sum_{k=0}^{n-3} B_{i, n-1}^{4}(t) B_{j, n-2}^{3}(t) B_{k, n-3}^{2}(t)\left\{\begin{array}{l}\left\{\left(\Delta b_{i} \otimes \Delta^{2} b_{j} \otimes \Delta^{3} b_{k}\right) \otimes \Delta b_{i} \otimes \Delta^{2} b_{j}\right\} \otimes \otimes \\ \left(\Delta b_{i} \otimes \Delta^{2} b_{j} \otimes \Delta^{3} b_{k}\right) \otimes \Delta b_{i}\end{array}\right\}\right\|\left\|\sum_{i=0}^{n-1} B_{i, n-1}(t) \Delta b_{i}\right\|^{2}}$,

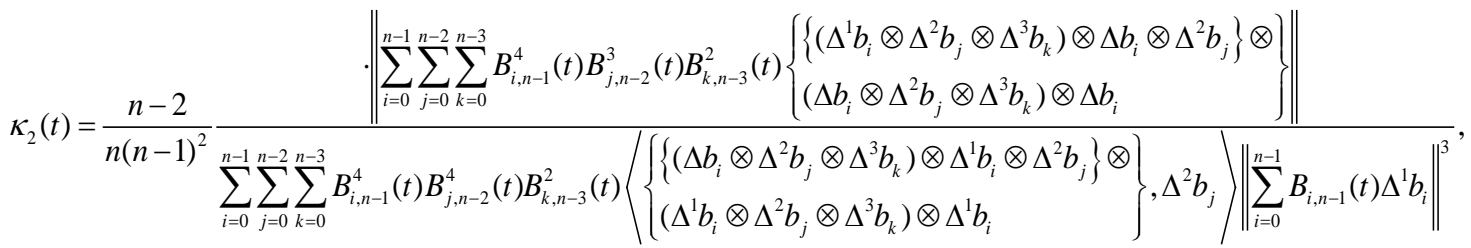

$\sum_{i=0}^{n-1} \sum_{j=0}^{n-2} \sum_{k=0}^{n-3} B_{i, n-1}^{2}(t) B_{j, n-2}^{2}(t) B_{k, n-3}^{2}(t)\left\langle\left\{\left(\Delta b_{i} \otimes \Delta^{2} b_{j} \otimes \Delta^{3} b_{k}\right) \otimes \Delta b_{i} \otimes \Delta^{2} b_{j}\right\}, \Delta^{3} b_{k}\right\rangle$

$\kappa_{3}(t)=\frac{(n-1)^{2}(n-3)}{n} \frac{\sum_{i=0}^{n-1} \sum_{j=0}^{n-2} \sum_{k=0}^{n-3} \sum_{m=0}^{n-4} B_{i, n-1}(t) B_{j, n-2}(t) B_{k, n-3} B_{m, n-4}(t)\left\langle\left(\Delta^{1} b_{i} \otimes \Delta^{2} b_{j} \otimes \Delta^{3} b_{k}\right), \Delta^{4} b_{m}\right\rangle\left\|\sum_{i=0}^{n-1} B_{i, n-1}(t) \Delta^{1} b_{i}\right\|}{\sum_{i=0}^{n-1} \sum_{j=0}^{n-2} \sum_{k=0}^{n-3} B_{i, n-1}^{2}(t) B_{j, n-2}^{2}(t) B_{k, n-3}^{2}(t)\left\langle\left\{\left(\Delta^{1} b_{i} \otimes \Delta^{2} b_{j} \otimes \Delta^{3} b_{k}\right) \otimes \Delta^{1} b_{i} \otimes \Delta^{2} b_{j}\right\}, \Delta^{3} b_{k}\right\rangle}$.

Proof If we write (14), (17) and (18) in (9), the proof of equation (20) is straightforward.The second curvature $\kappa_{2}(t)$ is obtained by writing $B_{1}(t), \kappa_{1}(t), P^{\prime \prime \prime}(t)$ and $\left\|P^{\prime}(t)\right\|^{3}=n^{3}\left\|\sum_{i=0}^{n-1} B_{i, n-1}(t) \Delta^{1} b_{i}\right\|^{3}$ in the equation (10). From the equation (2), we write the fourth derivative of $P(t)$ as follows: 


$$
P^{(4)}(t)=n(n-1)(n-2)(n-3) \sum_{i=0}^{n-4} B_{i, n-4}(t) \Delta^{4} b_{i}
$$

The third curvature $\kappa_{3}(t)$ is obtained writing the equations $B_{2}(t), \kappa_{1}(t), \kappa_{2}(t), P^{(4)}(t)$ and $\left\|P^{\prime}(t)\right\|^{4}=n^{4}\left\|\sum_{i=0}^{n-1} B_{i, n-1}(t) \Delta^{1} b_{i}\right\|^{4}$ in the equation (11).

Now, we shall investigate to the Serret-Frenet elements $\left\{T, N, B_{1}, B_{2}, \kappa_{1}, \kappa_{2}, \kappa_{3}\right\}$ of a Bézier curve with degree $n$ at starting point $t=0$ and ending point $t=1$ in Euclidean space $E^{4}$.

Corollary 3.3 Let $P(t)$ be a non-unit speed Bézier curve with control points $b_{0}, b_{1}, \ldots, b_{n}$ defined by the equation (12). For the starting point $t=0$, we have

$$
\begin{aligned}
& \left.T(t)\right|_{t=0}=\frac{\Delta b_{0}}{\left\|\Delta b_{0}\right\|}, \\
& \left.N(t)\right|_{t=0}=\frac{\left\{\left(\Delta b_{0} \times \Delta b_{1} \times \Delta b_{2}\right) \times \Delta b_{0} \times \Delta b_{1}\right\} \times\left(\Delta b_{0} \times \Delta b_{1} \times \Delta b_{2}\right) \times \Delta b_{0}}{\left\|\left\{\left(\Delta b_{0} \times \Delta b_{1} \times \Delta b_{2}\right) \times \Delta b_{0} \times \Delta b_{1}\right\} \times\left(\Delta b_{0} \times \Delta b_{1} \times \Delta b_{2}\right) \times \Delta b_{0}\right\|},
\end{aligned}
$$

or

$$
\begin{aligned}
& \left.N(t)\right|_{t=0}=-\frac{\Delta b_{0}}{\left\|\Delta b_{0}\right\|} \frac{\cos \alpha_{01}}{\left|\sin \alpha_{01}\right|}+\frac{\Delta b_{1}}{\left\|\Delta b_{1}\right\|} \frac{1}{\left|\sin \alpha_{01}\right|}, \\
& \left.B_{1}(t)\right|_{t=0}=\frac{\left(\Delta b_{0} \otimes \Delta b_{1} \otimes \Delta b_{2}\right) \otimes \Delta b_{0} \otimes \Delta b_{1}}{\left\|\left(\Delta b_{0} \otimes \Delta b_{1} \otimes \Delta b_{2}\right) \otimes \Delta b_{0} \otimes \Delta b_{1}\right\|},
\end{aligned}
$$

or

$$
\begin{aligned}
& \left.B_{1}(t)\right|_{t=0}=-\Delta^{1} b_{0} \frac{\left\|\Delta^{1} b_{1}\right\|\left\|\Delta^{1} b_{2}\right\| \sin \alpha_{12}}{\left\|\Delta^{1} b_{0} \otimes \Delta^{1} b_{1} \otimes \Delta^{1} b_{2}\right\|}-\Delta^{1} b_{1} \frac{\left\|\Delta^{1} b_{0}\right\|\left\|\Delta^{1} b_{2}\right\| \sin \alpha_{02}}{\left\|\Delta^{1} b_{0} \otimes \Delta^{1} b_{1} \otimes \Delta^{1} b_{2}\right\|}-\Delta^{1} b_{2} \frac{\left\|\Delta^{1} b_{0}\right\|\left\|\Delta^{1} b_{1}\right\| \sin \alpha_{01}}{\left\|\Delta^{1} b_{0} \otimes \Delta^{1} b_{1} \otimes \Delta^{1} b_{2}\right\|}, \\
& \left.B_{2}(t)\right|_{t=0}=\frac{\Delta b_{0} \otimes \Delta b_{1} \otimes \Delta b_{2}}{\left\|\Delta b_{0} \otimes \Delta b_{1} \otimes \Delta b_{2}\right\|}
\end{aligned}
$$

where $\alpha_{01}$ is the angle between $\Delta b_{0}$ and $\Delta b_{1}, \alpha_{02}$ is the angle between $\Delta b_{0}$ and $\Delta b_{2}, \alpha_{12}$ is the angle between $\Delta b_{1}$ and $\Delta b_{2}$.

Corollary 3.4 The first, second and third curvatures $\kappa_{1}, \kappa_{2}, \kappa_{3}$ of $P(t)$ defined by the equation (12) at starting point $t=0$ in Euclidean space $E^{4}$ are given by

$$
\begin{aligned}
& \left.\kappa_{1}(t)\right|_{t=0}=\frac{n-1}{n} \frac{\left\langle\left\{\left(\Delta b_{0} \times \Delta b_{1} \times \Delta b_{2}\right) \times \Delta b_{0} \times \Delta b_{1}\right\} \times\left(\Delta b_{0} \times \Delta b_{1} \times \Delta b_{2}\right) \times \Delta b_{0}, \Delta b_{1}\right\rangle}{\left\|\left\{\left(\Delta b_{0} \times \Delta b_{1} \times \Delta b_{2}\right) \times \Delta b_{0} \times \Delta b_{1}\right\} \times\left(\Delta b_{0} \times \Delta b_{1} \times \Delta b_{2}\right) \times \Delta b_{0}\right\|\left\|\Delta b_{0}\right\|^{2}} \\
& \left.\kappa_{2}(t)\right|_{t=0}=\frac{(n-2)}{n} \frac{\left\|\left\{\left(\Delta b_{0} \times \Delta b_{1} \times \Delta b_{2}\right) \times \Delta b_{0} \times \Delta b_{1}\right\} \times\left(\Delta b_{0} \times \Delta b_{1} \times \Delta b_{2}\right) \times \Delta b_{0}\right\|}{\left\|\left(\Delta b_{0} \times \Delta b_{1} \times \Delta b_{2}\right) \times \Delta b_{0} \times \Delta b_{1}\right\|\left\|\Delta b_{0}\right\|} \frac{\left\langle\left(\Delta b_{0} \times \Delta b_{1} \times \Delta b_{2}\right) \times \Delta b_{0} \times \Delta b_{1}, \Delta b_{2}\right\rangle}{\left\langle\left\{\left(\Delta b_{0} \times \Delta b_{1} \times \Delta b_{2}\right) \times \Delta b_{0} \times \Delta b_{1}\right\} \times\left(\Delta b_{0} \times \Delta b_{1} \times \Delta b_{2}\right) \times \Delta b_{0}, \Delta b_{1}\right\rangle} \\
& \left.\kappa_{3}(t)\right|_{t=0}=\frac{n-3}{n} \frac{\left\langle\Delta b_{0} \otimes \Delta b_{1} \otimes \Delta b_{2}, \Delta b_{3}\right\rangle}{\left\langle\left(\Delta b_{0} \otimes \Delta b_{1} \otimes \Delta b_{2}\right) \otimes \Delta b_{0} \otimes \Delta b_{1}, \Delta b_{2}\right\rangle} \frac{\left\|\left(\Delta b_{0} \otimes \Delta b_{1} \otimes \Delta b_{2}\right) \otimes \Delta b_{0} \otimes \Delta b_{1}\right\|}{\left\|\Delta b_{0} \otimes \Delta b_{1} \otimes \Delta b_{2}\right\|\left\|\Delta b_{0}\right\|}
\end{aligned}
$$


Corollary 3.5 Let $P(t)$ be a non-unit speed Bézier curve with control points $b_{0}, b_{1}, \ldots, b_{n}$ defined by the equation (12). For the ending point $t=1$, we have

$$
\begin{aligned}
& \left.T(t)\right|_{t=1}=\frac{\Delta b_{n-1}}{\left\|\Delta b_{n-1}\right\|}, \\
& \left.N(t)\right|_{t=1}=\frac{-\left\{\left(\Delta b_{n-3} \otimes \Delta b_{n-2} \otimes \Delta b_{n-1}\right) \otimes \Delta b_{n-1} \otimes \Delta b_{n-2}\right\} \otimes\left(\Delta b_{n-3} \otimes \Delta b_{n-2} \otimes \Delta b_{n-1}\right) \otimes \Delta b_{n-1}}{\left\|\left\{\left(\Delta b_{n-3} \otimes \Delta b_{n-2} \otimes \Delta b_{n-1}\right) \otimes \Delta b_{n-1} \otimes \Delta b_{n-2}\right\} \otimes\left(\Delta b_{n-3} \otimes \Delta b_{n-2} \otimes \Delta b_{n-1}\right) \otimes \Delta b_{n-1}\right\|}
\end{aligned}
$$

or

$$
\begin{aligned}
& \left.N(t)\right|_{t=1}=-\frac{\Delta b_{n-2}}{\left\|\Delta b_{n-2}\right\|} \frac{1}{\left|\sin \alpha_{(n-2)(n-1)}\right|}+\frac{\Delta b_{n-1}}{\| \Delta b_{n-1} \mid} \frac{\cos \alpha_{(n-2)(n-1)}}{\left|\sin \alpha_{(n-2)(n-1)}\right|}, \\
& \left.B_{1}(t)\right|_{t=1}=-\frac{\left(\Delta b_{n-3} \otimes \Delta b_{n-2} \otimes \Delta b_{n-1}\right) \otimes \Delta b_{n-1} \otimes \Delta b_{n-2}}{\left\|\left(\Delta b_{n-3} \otimes \Delta b_{n-2} \otimes \Delta b_{n-1}\right) \otimes \Delta b_{n-1} \otimes \Delta b_{n-2}\right\|},
\end{aligned}
$$

or

$$
\left.B_{1}(t)\right|_{t=1}=-\Delta b_{n-3} \frac{\left\|\Delta b_{n-2}\right\|\left\|\Delta b_{n-1}\right\| \sin \alpha_{(n-2)(n-1)}}{\left\|\Delta b_{n-3} \otimes \Delta b_{n-2} \otimes \Delta b_{n-1}\right\|}-\Delta b_{n-2} \frac{\left\|\Delta b_{n-1}\right\|\left\|\Delta b_{n-3}\right\| \sin \alpha_{(n-3)(n-1)}}{\left\|\Delta b_{n-3} \otimes \Delta b_{n-2} \otimes \Delta b_{n-1}\right\|}-\Delta b_{n-1} \frac{\left\|\Delta b_{n-3}\right\|\left\|\Delta b_{n-2}\right\| \sin \alpha_{(n-3)(n-2)}}{\left\|\Delta b_{n-3} \otimes \Delta b_{n-2} \otimes \Delta b_{n-1}\right\|},
$$

and

$$
\left.B_{2}(t)\right|_{t=1}=\frac{\Delta b_{n-3} \otimes \Delta b_{n-2} \otimes \Delta b_{n-1}}{\left\|\Delta b_{n-3} \otimes \Delta b_{n-2} \otimes \Delta b_{n-1}\right\|},
$$

where $\alpha_{(n-3)(n-2)}$ is the angle between $\Delta b_{n-3}$ and $\Delta b_{n-2}, \alpha_{(n-3)(n-1)}$ is the angle between $\Delta b_{n-3}$ and $\Delta b_{n-1}, \alpha_{(n-2)(n-1)}$ is the angle between $\Delta b_{n-2}$ and $\Delta b_{n-1}$.

Corollary 3.6 The first, second and third curvatures $\kappa_{1}, \kappa_{2}, \kappa_{3}$ of $P(t)$ defined by the equation (12) at ending point $t=1$ in Euclidean space $E^{4}$ are given by

$$
\begin{aligned}
\left.\kappa_{1}(t)\right|_{t=1}= & \frac{n-1}{n} \frac{\left\langle\left\{\left(\Delta b_{n-3} \otimes \Delta b_{n-2} \otimes \Delta b_{n-1}\right) \otimes \Delta b_{n-1} \otimes \Delta b_{n-2}\right\} \otimes\left(\Delta b_{n-3} \otimes \Delta b_{n-2} \otimes \Delta b_{n-1}\right) \otimes \Delta b_{n-1}, \Delta b_{n-2}\right\rangle}{\left\|\left\{\left(\Delta b_{n-3} \otimes \Delta b_{n-2} \otimes \Delta b_{n-1}\right) \otimes \Delta b_{n-1} \otimes \Delta b_{n-2}\right\} \otimes\left(\Delta b_{n-3} \otimes \Delta b_{n-2} \otimes \Delta b_{n-1}\right) \otimes \Delta b_{n-1}\right\|\left\|\Delta b_{n-1}\right\|^{2}},(39) \\
\left.\kappa_{2}(t)\right|_{t=1}= & \frac{(n-2)}{n} \frac{\left\|\left\{\left(\Delta b_{n-3} \otimes \Delta b_{n-2} \otimes \Delta b_{n-1}\right) \otimes \Delta b_{n-1} \otimes \Delta b_{n-2}\right\} \otimes\left(\Delta b_{n-3} \otimes \Delta b_{n-2} \otimes \Delta b_{n-1}\right) \otimes \Delta b_{n-1}\right\|}{\left\|\left(\Delta b_{n-3} \otimes \Delta b_{n-2} \otimes \Delta b_{n-1}\right) \otimes \Delta b_{n-1} \otimes \Delta b_{n-2}\right\|\left\|\Delta b_{n-1}\right\|} \\
& \cdot \frac{\left\langle\left(\Delta b_{n-3} \otimes \Delta b_{n-2} \otimes \Delta b_{n-1}\right) \otimes \Delta b_{n-1} \otimes \Delta b_{n-2}, \Delta b_{n-3}\right\rangle}{\left\langle\left\{\left(\Delta b_{n-3} \otimes \Delta b_{n-2} \otimes \Delta b_{n-1}\right) \otimes \Delta b_{n-1} \otimes \Delta b_{n-2}\right\} \otimes\left(\Delta b_{n-3} \otimes \Delta b_{n-2} \otimes \Delta b_{n-1}\right) \otimes \Delta b_{n-1}, \Delta b_{n-2}\right\rangle} \\
\left.\kappa_{3}(t)\right|_{t=1}= & -\frac{n-3}{n} \frac{\left\langle\Delta b_{n-3} \otimes \Delta b_{n-2} \otimes \Delta b_{n-1}, \Delta b_{n-4}\right\rangle}{\left\langle\left(\Delta b_{n-3} \otimes \Delta b_{n-2} \otimes \Delta b_{n-1}\right) \otimes \Delta b_{n-1} \otimes \Delta b_{n-2}, \Delta b_{n-3}\right\rangle} \frac{\left\|\left(\Delta b_{n-3} \otimes \Delta b_{n-2} \otimes \Delta b_{n-1}\right) \otimes \Delta b_{n-1} \otimes \Delta b_{n-2}\right\|}{\left\|\Delta b_{n-3} \otimes \Delta b_{n-2} \otimes \Delta b_{n-1}\right\|\left\|\Delta b_{n-1}\right\|}(41)
\end{aligned}
$$

\section{Conclusion}

In this work, Bézier curves are examined using the basic techniques of differential geometry. Serret-Frenet frame and curvatures of Bézier curves in Euclidean 4-space are formulated by the help of ternary product or triple vectorial product for Euclidean 4-space. These elements are calculated at each point, starting and ending point for the Bézier curves defined parametrically. These formulations provide an opportunity to determine the location of these curves in 
Euclidean 4-space. Thus, a different perspective is given to these curves, which were not studied in 4-dimensions in the literature.

\section{References}

[1] Forrest, A. R., "Curves and surfaces for computer-aided design”, University of Cambridge, United Kingdom, Cambridge, (1968).

[2] Anand, V. B., "Computer graphics and geometric modeling for engineers”, John Wiley and Sons Inc. (First Edition), New York, (1992).

[3] Farin, G., "Curves and surfaces for computer aided geometric design a practical guide”, Academic Press (Fourth Edition), San Diego, (1996).

[4] Marsh, D., “Applied geometry for computer graphics and CAD”, Springer-Verlag London Berlin Heidelberg (Second Edition), (2000).

[5] Kenmotsu, K. "Periodic mean curvature and Bézier curves. Differential geometry and related topics”, World Sci. Publ., River Edge, NJ, (2002) : 135-146.

[6] Georgiev, G. H. "On the shape of of the cubic Bézier curve. Pure and applied differential geometry_PADGE”, Ber. Math. Shaker Verlag, Aachen, (2007) : 98-106.

[7] Samanci, H. K., Celik, S., Incesu, M., “The Bishop Frame of Bezier Curves”, Life Science Journal, 12(6) (2015).

[8] Erkan, E., Yüce, S., "Serret-Frenet Frame and Curvatures of Bézier Curves”, Mathematics, 6(12) (2018) : 321-321.

[9] Aléssio, O., Düldül, M., Düldül B. U., Badr S. A., Abdel-All N. H., "Differential geometry of non-transversal intersection curves of three parametric hypersurfaces in Euclidean 4-space”, Comput. Aided Geom. Des., 31 (2014): 712-727.

[10] Williams, M. Z., Stein, F. M., “A triple product of vectors in four-space”, Math Mag, 37 (1964) : 230-235.

[11] Shaw, R., "Vector cross products in $n$ dimensions”, Int. J. Math. Educ. Sci. Technol., 18(6) (1987) : 803-816.

[12] Aléssio, O., "Differential geometry of intersection curves in $\mathbb{R}^{4}$ three implicit surfaces", Comput. Aided Geom. Des., 26 (2009) : 455-471. 